\title{
Longitudinal Monitoring of Lactate in Hospitalized and Ambulatory COVID-19 Patients
}

\author{
Thirumalaisamy P. Velavan, ${ }^{1,2 \star}$ Le Thi Kieu Linh, ${ }^{1,2}$ Andrea Kreidenweiss, ${ }^{1}$ Julian Gabor, ${ }^{1}$ Sanjeev Krishna, ${ }^{1,3,4}$ and \\ Peter G. Kremsner ${ }^{1,4}$ \\ ${ }^{1}$ Institute of Tropical Medicine, University of Tübingen, Tübingen, Germany; ${ }^{2}$ Vietnamese-German Center for Medical Research, Hanoi, Vietnam; \\ ${ }^{3}$ Institute for Infection Immunity, St George's University of London, London, United Kingdom; ${ }^{4}$ Centre de Recherches Médicales de Lambaréné,
} Lambaréné, Gabon

\begin{abstract}
Hypoxemia is readily detectable by assessing $\mathrm{SpO}_{2}$ levels, and these are important in optimizing COVID-19 patient management. Hyperlactatemia is a marker of tissue hypoxia, particularly in patients with increased oxygen requirement and microvascular obstruction. We monitored peripheral venous lactate concentrations in hospitalized patients with moderate to severe COVID-19 $(n=18)$ and in mild ambulatory COVID-19 patients in home quarantine $(n=16)$. Whole blood lactate decreased significantly during the clinical course and recovery in hospitalized patients $(P=0.008)$. The blood lactate levels were significantly higher in hospitalized patients than ambulatory patients (day 1: hospitalized versus ambulatory patients $P=0.002$; day 28: hospitalized versus ambulatory patients $P=\langle 0.0001$ ). Elevated lactate levels may be helpful in risk stratification, and serial monitoring of lactate may prove useful in the care of hospitalized COVID-19 patients.
\end{abstract}

Severe SARS-CoV-2 causes pneumonia and hypoxemia, often out of proportion to symptoms reported by COVID-19 patients. ${ }^{1}$ Hypoxemia is readily detectable by assessing $\mathrm{SpO}_{2}$ levels, and these are important in optimizing patient management. The degree to which systemic hypoxemia results in tissue hypoxemia and an increase in anaerobic glycolysis is unknown in COVID-19. Several factors could mitigate the tissue effects of decreased oxygen diffusion capacity in the lungs, including increased oxygen delivery by a right shift in the oxygen-hemoglobin dissociation curve (Bohr effect), increased fractional unloading of oxygen, and increased hemoglobin concentrations. Hyperlactatemia is one marker of tissue hypoxia, particularly when anaerobic tissue metabolism is increased in infections where there is anemia, fever with increased oxygen requirement, and microvascular obstruction. Severe malaria exemplifies these predispositions, and increased lactate levels ( $\geq 5 \mathrm{mmol} / \mathrm{L}$ ), particularly if they are sustained after admission, are one of the best prognostic indicators of disease severity and fatal outcomes. ${ }^{2}$ Lactate levels are also associated with poor clinical outcomes, as observed in a recent retrospective observational study in patients with COVID-19 pneumonia. ${ }^{3}$ We monitored peripheral venous lactate concentrations in hospitalized patients with moderate to severe COVID-19 $(n=18)$ and in mild ambulatory COVID-19 patients in home quarantine $(n=16)$.

The study was conducted in Germany. A total of 186 hospitalized patients were screened for inclusion, and 18 of them were enrolled in the hospitalized group. The hospitalized patients were more commonly elderly, with pre-existing medical conditions, and were on co-medications that cause adverse interactions when taken with hydroxychloroquine (HCQ). Hospitalized patients also showed comorbidities, especially diabetes (59/186; 32\%), renal insufficiency (53/186; $28 \%$ ), and heart failure (47/186; 25\%). Of the 186 patients examined, 168 could not be recruited for various reasons (Table 1). A detailed description of the inclusion and exclusion criteria for hospitalized

* Address correspondence to Thirumalaisamy P. Velavan, Institute of Tropical Medicine, University of Tübingen, Wilhelmstrasse 27, Tübingen 72074, Germany. E-mail: velavan@medizin.uni-tuebingen.de
COVID-19 patients is available at the European union (EU) Register of Clinical Studies (EudraCT-Number 2020-001224-33; https://www.clinicaltrialsregister.eu/ctr-search/trial/2020001224-33/DE).

A total of 21 ambulatory patients were screened, and 16 of those who met the inclusion criterion were enrolled. Invited to participate in the study were ambulatory patients who were classified as SARS-CoV2-PCR-positive by the local health department and showed no clinical complications other than fever and were quarantined at home. None of the ambulatory patient reported breathing difficulties or respiratory distress during recruitment. Of the 21 patients examined, five did not meet the inclusion criteria because of co-medication, low body weight, QT/QTc interval, and respiratory complications (Table 1). A detailed description of the inclusion and exclusion criteria for ambulatory COVID-19 patients is available in the EU Register of Clinical Studies (EudraCT Number: $2020001512-$ 26; https://www.clinicaltrialsregister.eu/ctr-search/trial/2020001512-26/DE).

The baseline patient characteristics of the COVID-19 patients are summarized in Table 2. Of the 18 hospitalized patients examined, the median age was 69 (range 28-83) years and $63 \%$ were male. Of the 16 examined ambulatory patients, the median age was 43 (range 18-63) years and $44 \%$ were male. Hospitalized patients were older than ambulatory patients $(P<0.001)$. Hospitalized patients were SARS-CoV2-positive on day 1 and negative on day 7 (except for four positives) on day 14, whereas all ambulatory patients were SARS-CoV-2-positive on day 1 and became negative on day 7 and day 14 (except for two). During the clinical course of COVID19 , one patient in the hospitalized group died (1/18), whereas others recovered. None of the ambulatory patients had developed any clinical complications. Kidney or respiratory failure, shock, or death was not observed in the ambulatory patients. Furthermore, none of the ambulatory patients were hospitalized nor required supplemental oxygen during the follow-up period.

Both hospitalized and ambulatory patients were followed up longitudinally. Serum samples were collected from hospitalized patients on days 1, 7, 14, and 28 and from ambulatory patients on days 1,14 , and 28. COVID-19 was diagnosed with a RealStar ${ }^{\circledR}$ SARS-CoV-2 real-time PCR targeting the S gene 
TABLE 1

Clinical characteristics of hospitalized and ambulatory COVID-19 patients during recruitment

\begin{tabular}{|c|c|c|}
\hline COVID-19 patient & Hospitalized (18/186) & Ambulatory (16/21) \\
\hline Patients screened & 186 & 21 \\
\hline Patients fulfill inclusion criteria & 18 & 17 \\
\hline Patients who did not consent/eligible to be included & 168 & 5 \\
\hline Exclusion criterion (as below) & 70 & 4 \\
\hline Severe cardiac failure & 1 & - \\
\hline Cardiological risk profile & 0 & 1 \\
\hline Delirium and QT prolongation & 1 & - \\
\hline Dementia & 15 & - \\
\hline Low body weight & 1 & 1 \\
\hline $\mathrm{HCQ}$ as co-medication & 4 & - \\
\hline ICU and respiratory failure or hemodynamic instability & 9 & - \\
\hline ICU and HCQ as co-medication & 1 & - \\
\hline ICU and QT prolongation & 2 & - \\
\hline Cognitive impairment & 2 & - \\
\hline Percutaneous endoscopic gastrostomy & 2 & - \\
\hline QT prolongation & 8 & 2 \\
\hline Hematological disease & 1 & - \\
\hline Hemato-oncological disease & 12 & - \\
\hline Hemato-oncological disease and dementia & 1 & - \\
\hline Hemato-oncological disease and cognitive impairment & 1 & - \\
\hline Hemato-oncological disease and myocardial infarction & 1 & - \\
\hline Hemato-oncological disease and QT prolongation & 1 & - \\
\hline Myasthenia gravis & 1 & - \\
\hline Retinopathy/macular degeneration & 3 & - \\
\hline Retinopathy/macular degeneration and bradyarrhythmia & 1 & - \\
\hline Retinopathy/macular degeneration and ICU & 1 & - \\
\hline Retinopathy/macular degeneration and QT prolongation and dementia & 1 & - \\
\hline
\end{tabular}

$\mathrm{HCQ}=$ hydroxychloroquine; $\mathrm{ICU}=$ intensive care unit.

of SARS-CoV-2 (Altona Diagnostics, Hamburg, Germany). Lactate concentrations (assayed with Sigma-Aldrich, Saint Louis, Missouri, on a PHOmo microplate reader, Autobio Diagnostics, Zhengzhou, China) were obtained on days 1, 7, 14 , and 28 in hospitalized patients, and on days 1, 14, and 28 in ambulatory patients. The nonparametric Friedman test was used to detect differences in the analysis of variance with repeated measurements (SPSS Statistics, IBM, Armonk, NY).
Median whole blood lactate levels in hospitalized patients were d1 $=3.3, \mathrm{~d} 7=2.6, \mathrm{~d} 14=2.2, \mathrm{~d} 28=1.9 \mathrm{mmol}$, and in ambulatory patients, $\mathrm{d} 1=1.4, \mathrm{~d} 14=1.4, \mathrm{~d} 28=1.5 \mathrm{mmol}$. Whole blood lactate decreased significantly during the clinical course and recovery in hospitalized patients $(P=0.008)$. The blood lactate levels were significantly higher in hospitalized patients than ambulatory patients (day 1: hospitalized versus ambulatory patients $P=0.002$; day 28: hospitalized versus ambulatory patients $P=<0.0001$ ).

TABLE 2

Baseline characteristics of hospitalized and ambulatory COVID-19 patients

\begin{tabular}{|c|c|c|c|}
\hline COVID-19 patient characteristic & Hospitalized $(n=18)$ & Ambulatory $(n=16)$ & $P$-value \\
\hline Median age (range) & $69(28-83)$ & $43(18-63)$ & $<0.0001$ \\
\hline Male $(n)(\%)$ & $12(63 \%)$ & $7(44 \%)$ & NS \\
\hline Median blood pressure (systolic/diastolic) & $135 / 74$ & $143 / 92$ & NS \\
\hline Median respiratory rate (breaths/minute) (SD) & $20( \pm 2.7)$ & $18( \pm 3.1)$ & NS \\
\hline Median heart rate (beats per minute) (SD) & $80( \pm 9.8)$ & $71( \pm 17.5)$ & NS \\
\hline \multicolumn{4}{|l|}{ Geometric mean (range minimum. - maximum) } \\
\hline Leukocyte $(n / \mu \mathrm{L})$ & $6,139(3,230-12,300)$ & NA & - \\
\hline Neutrophils (\%) & $67(36-82)$ & NA & - \\
\hline Lymphocytes (\%) & $19(8-49)$ & NA & - \\
\hline Monocytes (\%) & $9(2-14)$ & NA & - \\
\hline Eosinophils (\%) & $0(0-4)$ & NA & - \\
\hline Basophils (\%) & $0.5(0.1-1.3)$ & NA & - \\
\hline Erythrocytes (million/ $\mu \mathrm{L}$ ) & $4(3-6)$ & NA & - \\
\hline Hematocrit (\%) & $37(28-47)$ & NA & - \\
\hline $\mathrm{Hb}(\mathrm{g} / \mathrm{dL})$ & $12.7(8-16)$ & NA & - \\
\hline Thrombocytes $(1,000 / \mu \mathrm{L})$ & $233(105-572)$ & NA & - \\
\hline Fibrinogen $(\mathrm{mg} / \mathrm{dL})^{*}$ & $472(211-768)$ & NA & - \\
\hline Procalcitonin $(\mathrm{ng} / \mathrm{mL})$ & $0.09(0.01-2.56)$ & NA & - \\
\hline Creatine kinase (U/L) & $93(19-2,468)$ & NA & - \\
\hline AST (U/L) & $39(17-748)$ & NA & - \\
\hline ALT (U/L) & $31(9-401)$ & NA & - \\
\hline $\mathrm{LDH}(\mathrm{U} / \mathrm{L})$ & $295(153-899)$ & NA & - \\
\hline
\end{tabular}

AST = aspartate Aminotransferase; ALT = alanine aminotransferase; $\mathrm{LDH}$ = lactate dehydrogenase; NA = not available; NS = nonsignificant as mild COVID-19 was observed in ambulatory patients without clinical complications.

${ }^{*}$ Fibrinogen levels not available for one hospitalized patient. 


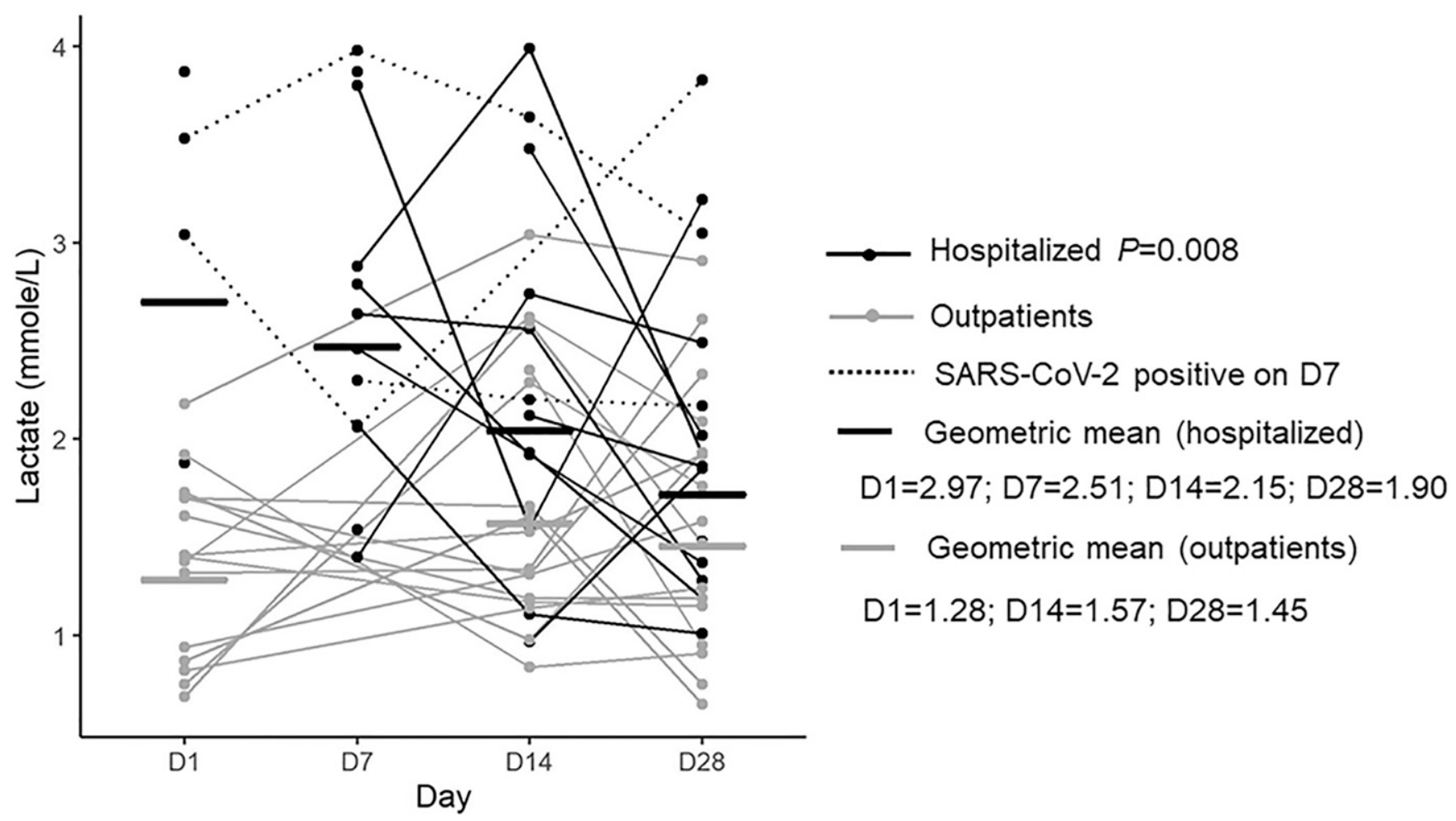

FIGURE 1. Lactate levels associated with COVID-19 clinical phenotype (hospitalized patients vs. outpatients). The nonparametric Friedman test was used to detect differences in the analysis of variance with repeated measurements. Figures were created using ggplot2 package version 3.2.1 supported on R program software version 3.5.0.

Lactate levels in patients with COVID-19 were significantly higher in hospitalized patients with more severe disease than ambulatory patients having mild disease, with levels declining over weeks in hospitalized patients. At day 28, lactate levels were comparable between hospitalized patients and ambulatory patients. Four hospitalized patients with SARS-CoV-2 virus positivity on day 7 took a longer time to clear the virus than other hospitalized patients and had consistently elevated lactate levels (Figure 1).

Lactate levels in our hospitalized patients were moderately elevated compared with pneumonia caused by non-SARSCoV-2 infections, where more severe disease (CURB-65 $\geq 4$ ) had a median lactate level of around $4 \mathrm{mmol} / \mathrm{L} .{ }^{4}$ In severe systemic infections such as malaria, admission lactate levels are also elevated with cutoffs $>5 \mathrm{mmol} / \mathrm{L}$ associated with severe disease and fatal outcomes, particularly if sustained for hours. The time course of decreases in lactate levels in COVID19 reflects a longer course of illness. In some patients, the lactate level increased at D28, perhaps reflecting increased activity when diffusion defects in lungs may not have recovered fully. Elevations in lactate levels reflect different pathophysiological processes with decreased oxygen diffusion in the lungs of patients with COVID-19 pneumonia, perhaps with adequate tissue oxygenation being maintained in many patients until the disease progresses in severity ("silent hypoxia"), 5,6 whereas in malaria, tissue hypoxia caused by microvascular obstruction increases lactate production in the host tissues. ${ }^{7}$

A total of $10 \%$ of hospitalized patients could only be recruited, as many of the hospitalized patients do not fulfill the inclusion criteria, that includes denial of informed written consent. This is acknowledged as a major limitation in this study. Although age and concurrent comorbidities of COVID19 patients largely determine the COVID-19 clinical course, ${ }^{8,9}$ elevated lactate levels equally facilitate the assessment of disease course.

Received September 30, 2020. Accepted for publication January 4, 2021.

Published online January 11, 2021.

Acknowledgments: We acknowledge the support of the study team and Carlos Lamsfus Calle for the technical support with the figure. Publication charges for this article were waived due to the ongoing pandemic of COVID-19.

Financial support: This work was funded by the Federal Ministry of Education and Research (BMBF) (BMBF-01KI2052) and the Federal Ministry of Health (BMG) (BMG-ZMVI1-1520COR801).

Disclosure: All related data supporting the results reported in the article is available within the manuscript. T. P. V. is a member of the Pan African Network for Rapid Research, Response, and Preparedness for Infectious Diseases Epidemics consortium (PANDORA-ID-NET).

Authors' addresses: Thirumalaisamy P. Velavan, Le Thi Kieu Linh, Andrea Kreidenweiss, and Julian Gabor, Institute of Tropical Medicine, University of Tübingen, Tübingen, Germany, E-mails: velavan@ medizin.uni-tuebingen.de, Ik.linh1509@gmail.com, andrea.kreidenweiss@ uni-tuebingen.de, and julian.gabor@uni-tuebingen.de. Sanjeev Krishna, Infection and Immunity, St. George's University of London, London, United Kingdom, E-mail: s.krishna@sgul.ac.uk. Peter G. Kremsner, Department of Parasitology, Institute of Tropical Medicine, University of Tübingen, Tübingen, Germany, E-mail: peter.kremsner@unituebingen.de.

This is an open-access article distributed under the terms of the Creative Commons Attribution (CC-BY) License, which permits unrestricted use, distribution, and reproduction in any medium, provided the original author and source are credited. 


\section{REFERENCES}

1. Berezin $L$ et al., 2020. The diagnostic accuracy of subjective dyspnea in detecting hypoxemia among outpatients with COVID-19. medRxiv. Available at: https://doi.org/10.1101/ 2020.08.10.20172262.

2. Kremsner PG et al., 2009. Prognostic value of circulating pigmented cells in African children with malaria. J Infect Dis 199: 142-150.

3. Vassiliou AG et al., 2020. Lactate kinetics reflect organ dysfunction and are associated with adverse outcomes in intensive care unit patients with COVID-19 pneumonia: preliminary results from a Greek single-centre study. Metabolites 10: E386.

4. Chen YX, Li CS, 2015. Lactate on emergency department arrival as a predictor of mortality and site-of-care in pneumonia patients: a cohort study. Thorax 70: 404-410.
5. Fuglebjerg NJU, Jensen TO, Hoyer N, Ryrso CK, Lindegaard B, Harboe ZB, 2020. Silent hypoxia in patients with SARS CoV-2 infection before hospital discharge. Int $J$ Infect Dis 99: 100-101.

6. Xie J, Covassin N, Fan Z, Singh P, Gao W, Li G, Kara T, Somers VK, 2020. Association between hypoxemia and mortality in patients with COVID-19. Mayo Clin Proc 95: 1138-1147.

7. Agbenyega T, Angus BJ, Bedu-Addo G, Baffoe-Bonnie B, Guyton T, Stacpoole PW, Krishna S, 2000. Glucose and lactate kinetics in children with severe malaria. J Clin Endocrinol Metab 85: 1569-1576.

8. Velavan TP, Meyer CG, 2020. Mild versus severe COVID-19: laboratory markers. Int $\mathrm{J}$ Infect Dis 95: 304-307.

9. Velavan TP, Meyer CG, 2020. The COVID-19 epidemic. Trop Med Int Health 25: 278-280. 